\title{
Article
}

\section{The effect of caffeine mouth rinse on self- paced cycling performance}

Bottoms, Lindsay, Hurst, Howard Thomas, Scriven, A, Lynch, F, Bolton, J, Vercoe, L., Shone, Z., Barry, G. and Sinclair, Jonathan Kenneth

Available at http://clok.uclan.ac.uk/13684/

Bottoms, Lindsay, Hurst, Howard Thomas ORCID: 0000-0001-7889-8592, Scriven, A, Lynch, F, Bolton, J, Vercoe, L., Shone, Z., Barry, G. and Sinclair, Jonathan Kenneth ORCID: 0000-0002-2231-3732 (2014) The effect of caffeine mouth rinse on self-paced cycling performance. Comparative Exercise Physiology, 10 (4). pp. 239-245. ISSN 1755-2540

It is advisable to refer to the publisher's version if you intend to cite from the work. http://dx.doi.org/10.3920/CEP140015

For more information about UCLan's research in this area go to http://www.uclan.ac.uk/researchgroups/ and search for <name of research Group>.

For information about Research generally at UCLan please go to http://www.uclan.ac.uk/research/

All outputs in CLoK are protected by Intellectual Property Rights law, including Copyright law. Copyright, IPR and Moral Rights for the works on this site are retained by the individual authors and/or other copyright owners. Terms and conditions for use of this material are defined in the policies page.

\section{CLoK}

Central Lancashire online Knowledge www.clok.uclan.ac.uk

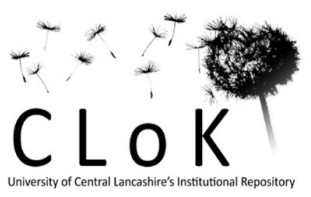




\section{The effect of caffeine mouth rinse on self paced cycling performance}

2

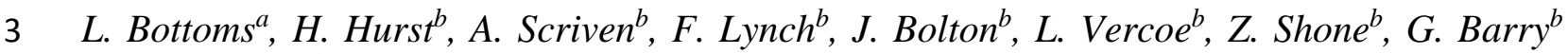
4 and J. Sinclair ${ }^{b}$

5

6 a School of Health, Sport and Bioscience, University of East London, Water Lane, Stratford, 7 UK

$8{ }^{b}$ Division of Sport Exercise and Nutritional Sciences, University of Central Lancashire, Fylde 9 Road, Preston, UK

10

11 Corresponding Author:

12 Dr Lindsay Bottoms,

13 School of Health, Sport and Bioscience,

14 University of East London,

15 Water Lane,

16 Stratford,

17 UK

18 E15 4LZ

19 Tel: 02082283371

20 Email: L.Bottoms@uel.ac.uk

21

22

23

24 


\section{Abstract}

The aim of the study was to determine whether caffeine mouth rinse would improve 30 minutes self-paced cycling trial. Twelve healthy active males (age $20.5 \pm 0.7$ yrs, mass 87.4 $\pm 18.3 \mathrm{~kg}$ ) volunteered for the study. They attended the laboratory on 3 separate occasions performing a 30 minute self-paced cycling trial. On one occasion water was given as a mouth rinse for $5 \mathrm{~s}$ (PLA), on another occasion a 6.4\% CHO solution was given for $5 \mathrm{~s}$ and finally a caffeine solution (containing $32 \mathrm{mg}$ of caffeine dissolved in $125 \mathrm{ml}$ water; CAF) was given for $5 \mathrm{~s}$. Distance cycled, heart rate, ratings of perceived exertion, cadence, speed and power output were recorded throughout all trials. Distance cycled during the CAF mouth rinse trial $(16.2 \pm 2.8 \mathrm{~km})$ was significantly greater compared to PLA trial $(14.9 \pm 2.6 \mathrm{~km})$. There was no difference between $\mathrm{CHO}$ and CAF trials $(\mathrm{P}=0.89)$. Cadence, power and velocity were significantly greater during the CAF trial compared to both PLA and CHO $(\mathrm{P}<0.05)$. There were no differences between trials for HR and RPE $(\mathrm{P}>0.05)$. Caffeine mouth rinse improves 30 minute cycling performance by allowing the participant to increase cadence, power and velocity without a concurrent increase in perceived exertion and heart rate.

\section{Key words: carbohydrate, oral receptors, ergogenic}


Caffeine has been unequivocally shown to improve cycling endurance performance either by prolonging time to exhaustion (Graham et al., 1998; Van Soeren \& Graham, 1998) or by decreasing time to complete set distances (Bridge \& Jones, 2006). In fact, very few research studies have found caffeine to have no effect on aerobic performance (Roelands et al., 2011). Although caffeine has been shown to improve endurance performance, the exact mechanism by which this is achieved remains unknown. Caffeine has been found to counter the effects of adenosine, which is a compound similar to caffeine (Davis \& Green, 2009). As such, caffeine is believed to enhance motor unit recruitment, bronchodilation, vasodilation, arousal, neuro-excitability, catecholamine secretion, lypolysis, plus reduce sleep and pain perception (Astorino \& Roberson, 2010; Beck et al., 2008; Hendrix et al., 2010; Hudson et al., 2008; Sokmen et al., 2008; Warren et al., 2010; Woolf et al., 2008).

The dampened pain perception causes an ergogenic effect on performance, via greater exercise duration (Beck et al., 2008; Bruce et al., 2000). Davis \& Green (2009) propose that performance decrements correlate with increases in muscle pain and a reduction in motor unit recruitment. However, Sokmen et al. (2008), Davis \& Green, 2009 and Warren et al. (2010) advocate that pain perception does not influence muscular performance; rather, improvements in performance are mediated through maintenance of the $\mathrm{Na}+/ \mathrm{K}+$ gradient and increases in calcium ions allowing more forceful contractions to occur and preventing plasma $\mathrm{K}+$ to rise. Caffeine also promotes the release of calcium ions from the sarcoplasmic reticulum, which ultimately allows more muscular contractions to take place, increasing strength and muscular endurance (Bellar et al., 2011; Jacobson et al., 1992; Warren et al., 2010). Conversely, Davis \& Green (2009) state that the concentrations of caffeine required to elicit this effect on the sarcoplasmic reticulum would be toxic to humans. In a recent review by Meeusen et al. (2013) they suggest that the main mechanism of action of caffeine is through antagonism of adenosine receptors, influencing the dopaminergic and other neurotransmitter systems. Adenosine and dopamine act on the brain and can influence factors such as motivation (Meeusen et al., 2013) and therefore this may be a large factor in the improvement of endurance performance with caffeine ingestion.

Previous research has shown that the optimum time for complete caffeine absorption is between 15 and 120 minutes post ingestion (Blanchard \& Sawers, 1983; Bonati et al., 1982; Kamimori et al., 1995; Kamimori et al., 2000) therefore researchers have often tested performance 1 hour post ingestion (Ryan et al., 2013). However, research has shown that absorption at the mouth is much more rapid and can produce quicker response to caffeine ingestion than capsule ingestion (Kamimori et al., 2002). This observation led researchers to use caffeine chewing gum to improve cycling performance with positive effects (Ryan et al., 2013; Paton et al., 2010). Caffeine can be absorbed through the buccal mucoa and therefore does not appear to require ingestion in order to produce ergogenic benefits (Nicolazzo et al., 2003; Thakur et al., 2007). Caffeine could then potentially increase performance by decreasing perceived exertion and reducing pain perception as mentioned previously as potential mechanisms for the ergogenic effect. Other mechanisms require a longer period of time for absorption therefore performance improvements are most likely pain perception and perceived exertion.

Carbohydrate mouth rinsing has been shown to improve high intensity cycling performance (Sinclair et al., 2014; Chambers et al., 2009; Pottier et al., 2010; Rollo et al., 2008) and is thought to improve performance through carbohydrate mouth receptors which control central 
mechanisms associated with motivation (Chambers et al., 2009). As the presence of caffeine receptors in the oral cavity is now established it could be hypothesised that a caffeine mouth rinse will also improve self paced cycling performance. Recent work by Beaven et al. (2013) has shown that a $1.2 \%$ caffeine mouth rinse solution improved repeated sprint performance which further supports the notion that caffeine mouth rinsing could improve high intensity cycling performance. However, more recent work of Doering et al. (2014) observed no improvements in time trial cycling performance when mouth rinsing $35 \mathrm{mg}$ of caffeine for 10 s, nor was there an increase in plasma caffeine concentrations. These conflicting results show that further research is needed. Therefore the aim of the current investigation was to determine whether caffeine mouth rinse improves 30 minute cycling time trial performance and whether there is a difference compared to a carbohydrate mouth rinse.

\section{Materials and Methods}

\section{Participants}

Twelve male participants (age $20.5 \pm 0.7$ yrs, height $170.5 \pm 18.8 \mathrm{~cm}$, mass $87.4 \pm 18.3 \mathrm{~kg}$ ) were recruited for this investigation. Participants were recreationally trained cyclists and free from musculoskeletal pathology at the time of data collection. All participants also provided written informed consent. The procedure utilised for this investigation was approved by the University of Central Lancashire, School of Sport Tourism and Outdoors, ethical committee.

\section{Procedure}

Data collection involved four laboratory sessions. Participants were familiarized with the experimental procedure in session 1, whereas sessions 2-4 were utilized for data collection. Participants completed 30 minute simulated time trials for maximum distance using a cycle ergometer (Monark Ergomedic 874E, Monark Exercise, AB, Varberg, Sweden). For sessions 2-4 in which experimental data was collected participants were administered either $25 \mathrm{ml}$ of a tasteless $6.4 \%$ maltodextrin (Maltodextrin, My Protein) solution (CHO), $0.032 \%$ caffeine (My Protein; this was selected as being the concentration of caffeine found typically in commercially available caffeinated drinks) solution (CAF) or a water bolus (PLA) which were rinsed for $5 \mathrm{~s}$ at each 6 minute interval of the cycling time trial in accordance with the overall time intervals utilised by Sinclair et al. (2014). This study utilized a blinded counterbalanced design, and each session was separated by 7 days.

\section{Visit 1}

This session represented a familiarization visit during which participants completed a 30 min time-trial in the same manner as the experimental conditions. From this session ergonomic aspects such as seat height and ergometer resistance could be obtained and maintained during data collection. In accordance with Sinclair et al. (2014) a resistance of $2.0 \mathrm{~kg}$ was selected which was deemed to be adequate and achievable for all participants at a cadence of 60 revs. $\min ^{-1}$.

\section{Visits 2-4}

Participants were examined 4 hours post prandial and had not consumed any alcohol/ caffeine or conducted any vigorous exercise in previous 24 hours prior to the commencement of data 
collection. Immediately preceding data collection all participants were fitted with a heart rate monitor (Polar RS100, Polar Electro), and then asked to position themselves in a comfortable position on the cycle ergometer. Prior to the data collection procedure a standardized warmup was conducted which consisted of 5 min of cycling using a resistance of $50 \mathrm{~W}$ in agreement with the warm up protocol utilized by Sinclair et al. (2014) for the same protocol. Data collection was conducted at the same time of day to avoid natural fluctuations in physiological parameters due to variations in circadian rhythmicity.

The cycling ergometer was connected to a computer using Monark software (Varberg, Sweden) in which the outcome measures of heart rate (HR), cadence (rev. $\mathrm{min}^{-1}$ ), power output (W) and distance covered (km) were obtained at 6 min intervals throughout the trials. In addition, participants were also required to state their perceived exertion (RPE) using the 6 to 20 point Borg scale (Borg, 1982) also at 6 min intervals. No interaction beyond requests for RPE and administration of the appropriate mouth rinse occurred between researchers and participants.

\section{Mouth rinse administration}

Each participant was given a $25 \mathrm{ml}$ bolus of a tasteless CHO, CAF or PLA for every 6 min of the total protocol. Participants rinsed the fluid around their mouths for $5 \mathrm{~s}$, and then spat the fluid back into a bowl.

\section{Statistical analyses}

Descriptive statistics of means \pm standard deviation were obtained for each condition. To compare total distance covered using the three solutions during the $30 \mathrm{~min}$ protocol a oneway repeated measures ANOVA was conducted. To examine any effects of mouth rinse on pacing, HR and RPE 5 x 3 (time x trial) repeated measures ANOVA's were also conducted Statistical significance was accepted at the $p \leq 0.05$ level. If the sphericity assumption was violated then the degrees of freedom were adjusted using the Greenhouse-Geisser correction. Effect sizes were calculated using and $\operatorname{Eta}^{2}\left(\eta^{2}\right)$. All statistical procedures were conducted using SPSS v20.0 (SPSS Inc., Chicago, IL, USA).

\section{Results}

Distance cycled:

@@@ FIGURE I NEAR HERE @@@ Figure I: Mean ( \pm SD) distance completed in 30 minutes during each condition $(\mathrm{n}=12)$. * denotes significant difference from PLA.

There was a main effect for distance $\left(P<.01, \eta^{2}=.51\right)$. Distance cycled during the CAF mouth rinse trial $(16.2 \pm 2.8 \mathrm{~km})$ was significantly greater compared to the PLA trial (14.9 $\pm 2.6 \mathrm{~km} ; P<.01)$ (Figure I). Distance cycled during the CHO trial $(15.9 \pm 2.9 \mathrm{~km})$ was also significantly greater than the PLA trial $(P=.03)$. There was no significant difference between CAF and CHO $(P=.90)$. However, 10 out of 12 participants cycled further during the CAF trial compared to $\mathrm{CHO}$, and 11 cycled further during the CAF trial compared to the PLA. 
Pacing:

Table I: Mean $( \pm \mathrm{SD})$ overall values for HR, RPE, cadence, power and speed for each condition $(n=12)$.

\begin{tabular}{llll}
\hline Mean ( \pm SD) & Placebo & CHO & CAF \\
\hline $\begin{array}{l}\text { Cadence (RPM) } \\
\text { Speed (km.h }\end{array}$ & $72.3 \pm 12.5$ & $77.0 \pm 13.7^{*}$ & $77.6 \pm 13.6^{*}$ \\
Power Output (W) & $30.0 \pm 5.4$ & $32.3 \pm 5.6^{*}$ & $32.3 \pm 5.9^{*}$ \\
Heart Rate (beats.min & $145.3 \pm 23.5$ & $153.3 \pm 29.0$ & $\left.155.2 \pm 27.5^{*}\right)$ \\
RPE (Borg Scale) & $160 \pm 26$ & $162 \pm 24$ & $156 \pm 24$ \\
\hline
\end{tabular}

*denotes significant difference from placebo.

Table I illustrates the mean overall values for each rinse condition. As can be seen in Figure IIa, there was a main effect for time for cadence $\left(P<.01, \eta^{2}=.49\right)$ with post hoc analysis showing cadence being significantly greater during the last 6 minutes of the trial $(P=.04)$. There was a main effect for trial, therefore mouth rinse had an effect on cadence $\left(P=.01, \eta^{2}=\right.$ .34 ), with CAF ( $80 \pm 17$ rev. $\mathrm{min}^{-1}$ ) producing a significantly greater cadence than PLA (74 \pm 17 rev.min $\left.{ }^{-1} ; \mathrm{P}=.03\right)$ with no difference to $\mathrm{CHO}\left(77 \pm 17\right.$ rev. $\left.\mathrm{min}^{-1} ; \mathrm{P}=.65\right)$. Speed also increased during the last 6 minutes of the trial (main effect for time; $P<.01, \eta^{2}=.40$ ). There was a main effect for trial $\left(P=.02, \eta^{2}=.29\right)$ with CAF mouth rinse producing a significantly greater speed (35.1 $\left.\pm 8.3 \mathrm{~km} . \mathrm{hr}^{-1}\right)$ than PLA (31.1 $\pm 7.6 \mathrm{~km} . \mathrm{hr}^{-1} ; P<.01$; Figure IIb). There was no difference between CAF and CHO $(\mathrm{P}=.57)$ and between CHO and PLA $(\mathrm{P}=.10)$. There was a main effect for time $\left(P<.01, \eta^{2}=.49\right)$ with power being greater during the last 6 minutes of the trial $(\mathrm{P}=.03)$. There was also an effect of trial $\left(P=0.01, \eta^{2}=.34\right)$ with CAF producing the greatest power output $(161 \pm 34 \mathrm{~W})$ compared to PLA $(148 \pm 33 \mathrm{~W} ; \mathrm{P}<.01)$.

@@@ FIGURE II NEAR HERE@@@ Figure II: Mean ( \pm SD) cadence (a) and speed (b) during the 30 minute exercise for each condition $(n=12)$.

\section{Heart rate and $R P E$}

HR increased throughout all trials with a main effect for time $\left(\mathrm{P}=.00, \eta^{2}=.79\right.$; Figure III) averaging at 160 $\pm 26,162 \pm 24$ and $156 \pm 24$ beats. $\mathrm{min}^{-1}$ for PLA, CHO and CAF respectively (Table I). There were no differences between trials $\left(P=0.15, \eta^{2}=.16\right)$. RPE increased with exercise duration with a main effect for time $\left(P<0.01, \eta^{2}=.93\right)$. There was also no difference between trials $\left(P=0.65, \eta^{2}=.04\right.$; Table I). 
@@@FIGURE III NEAR HERE@@@ Figure III: Mean ( \pm SD) heart rate (a) and RPE (b) during 30 minute exercise in each condition $(n=12)$.

\section{Blinding efficacy}

For the CAF rinse trial 5 out of 12 participants correctly identified that they were on a performance enhancing solution, for the CHO rinse trial 5 out of 12 identified the performance enhancing solution. Finally 7 out of 12 guessed the placebo solution correctly.

\section{Discussion}

The aim of the current study was to determine whether caffeine mouth rinse improved 30 minute cycling time trial performance and whether there was a difference compared to a CHO mouth rinse. This study represents only the second study to examine the ergogenic effect of caffeine mouth rinsing on cycling time trial performance.

The results demonstrated both caffeine and CHO mouth rinse increased distance cycled during 30 minutes of self-selected paced cycling. This supports previous observations in that carbohydrate mouth rinse improved high intensity performance (Sinclair et al., 2014; Chambers et al., 2009; Pottier et al., 2010; Rollo et al., 2008). The results also support those of Beaven et al. (2013) who found 1.2\% caffeine mouth rinse improved repeated sprint performance. However, the results conflicted with Doering et al. (2014) who found no improvement in cycling time trial performance with caffeine mouth rinse. These are the only previous research to have investigated caffeine mouth rinse on exercise performance.

Beaven et al. (2013) demonstrated that $1.2 \%$ caffeine mouth rinse improved repeated sprint performance. The present study examined a $0.032 \%$ caffeine solution as this is the quantity commonly found in commercially available caffeinated drinks. Studies investigating the effect of caffeine chewing gum on exercise performance (Ryan et al., 2013; Paton et al., 2010) used similar quantities (300mg and 240mg respectively) to that of the present study (128mg). Unfortunately, the different mode of exercise and the concentrations of caffeine make cross comparisons between these studies difficult. However, it is recommended that future research could be performed to determine whether there is a dose response to performance. Since caffeine is absorbed through the buccal mucosa (Nicolazzo et al., 2003; Thakur et al., 2007) it could be hypothesized that absorption is positively correlated with the concentration of caffeine that is present in the rinse solution which would produce and enhanced ergogenic effect. However, as previously mentioned Doering et al. (2014) observed no increases in plasma caffeine concentrations, so may be mouth rinsing will not produce a dose response due to absorption. The ergogenic effect could be due to receptors detecting caffeine in the mouth, rather than absorption similar to $\mathrm{CHO}$ rinsing. Recent research by Sinclair et al. (2014) demonstrated that 10 second CHO mouth rinse produced a greater performance enhancement than 5 seconds. This could be similar for caffeine mouth rinse suggesting that more caffeine activates more receptors in the mouth the longer the mouth rinse. 
248 The mechanism of action of caffeine is most likely to be adenosine antagonism (Meeusen et al., 2013). This then influences the dopaminergic and other neurotransmitter systems. In the present study there was no differences observed in RPE between trials, even though distance covered was greater during the caffeine trial as was power, speed and cadence. This suggests that the participants were able to perform at a greater intensity at a similar RPE, indicating that there was an increase in motivation with caffeine ingestion. The increase in motivation is thought to be a result of adenosine and dopamine acting on the brain following antagonism of the adenosine receptors (Meeusen et al., 2013). Improvement in performance may also be a result of a reduction in pain perception which is also thought to be one of caffeine's' ergogenic benefits (Davis \& Green, 2009). Chambers et al. (2009) investigated functional magnetic resonance imaging (fMRI) during carbohydrate mouth rinsing and determined that a $\mathrm{CHO}$ mouth rinse enhanced motivation and activity of motor control centres of the brain. It would of interests to both physiological and neurological populations to repeat this study using a caffeine mouth rinse to determine whether similar areas of the brain were stimulated.

The key practical implication of this research is that athletes/active individuals involved in moderate to high intensity exercise can use $\mathrm{CHO}$ and CAF mouths rinses instead of ingesting these solutions and still achieve meaningful physiological benefits. It appears based on the current findings that a CAF mouth rinse will mediate greater ergogenic improvements in comparison to $\mathrm{CHO}$; combining the two may improve performance to a greater extent as suggested by Beaven et al. (2013). Furthermore, the ingestion of both CAF and CHO has been associated with gastrointestinal distress during high intensity exercise as such the observations from the current investigation may have implications for the reduction of discomfort during exercise as rinsing the solution around the mouth does not require ingestion but still appears to provide ergogenic benefits.

In conclusion, the current investigation provides an addition to the current knowledge regarding the influence of both $\mathrm{CHO}$ and $\mathrm{CAF}$ mouth rinse on exercise performance and provides evidence to suggest that both $\mathrm{CHO}$ and $\mathrm{CAF}$ rinse can improve moderate to high intensity cycling performance. The underlying mechanisms behind these improvements in performance with the absence of solution ingestion remain undetermined currently and future work is required to determine the physiological processes that produce these performance enhancements. Nonetheless, this study shows that athletes performing in short duration cycling events could improve their overall performance by a CHO of CAF mouth rinse.

\section{References}

1. Astorino, T.A. and Roberson, D.W. 2010. Efficacy of Acute Caffeine Ingestion for Short-term High-Intensity Exercise Performance: A Systematic Review. Journal of Strength and Conditioning Research, 24: 257-265. 
2. Beaven, C.M., Maulder, P., Pooley, A., Kilduff. L. and Cook, C. 2013. Effects of caffeine and carbohydrate mouth rinses on repeated sprint performance. Appl Physiol Nutr Metab, 38(6): 633-7.

3. Beck, T. W., Housh, T.J., Malek, M.H., Mielke, M. and Hendrix, R. 2008.The Acute Effects of a Caffeine-Containing Supplementation on Bench Press Strength and Time to Running Exhaustion. Journal of Strength and Conditioning Research, 22(5): 16541658.

4. Bellar, D., Kamimori, G.H. and Glickman, E.L. 2011.The Effects of Low-Dose Caffeine on Perceived Pain During a Grip-to-Exhaustion Task. Journal of Strength and Conditioning Research, 25(5): 1225-1228.

5. Blanchard, J. and Sawers, S. J.A. 1983. The absolute bioavailability of caffeine in man. Eur. J. clin.Pharmac, 24: 93-98.

6. Bonati, M., Latini, R., Galletti, F., Young, J.F., Tognoni, G. and Garattini, S. 1982. Caffeine disposition after oral doses. Clin Pharmacol Ther, 32: 98-106.

7. Borg, G. 1982. Psychophysical bases of perceived exertion. Medicine \& Science In Sports \& Exercise, 14 (5): 377-381.

8. Bridge, C.A. and Jones, M.A. 2006. The effect of caffeine ingestion on $8 \mathrm{~km}$ run performance in a field setting. J. Sports Sci, 24(4): 433-9.

9. Bruce, C.L., Anderson, M.E., Fraser, S.F., Stepto, N.K., Klein, R., Hopkins ,W.G. and Hawley, J.A. 2000. Enhancement of 2000-m Rowing Performance After Caffeine Ingestion. Medicine \& Science in Sports \& Exercise, 32: 1958 - 1963.

10. Chambers, E.S., Bridge, M.W. and Jones, D.A. 2009. Carbohydrate sensing in the human mouth: effects on exercise performance and brain activity. Journal of Physiology, 587: 1779-1794.

11. Davis, J. K. and Green, J.M. 2009. Caffeine and Anaerobic Performance Ergogenic Value and Mechanisms of Action. Sports Medicine, 39(10): 813-832.

12. Doering, T.M., Fell, J.W., Leveritt, M.D., Desbrow, B., Shing, C.M. 2014. The effect of a caffeinated mouth-rinse on endurance cycling time-trial performance. Int J Sport Nutr Exerc Metab, 24(1):90-7.

13. Graham, T.E., Hibbert, E. and Sathasivam, P. 1998. Metabolic and exercise endurance effects of coffee and caffeine ingestion. J Appl Physiol, 85: 883-889.

14. Hendrix, C.R., Housh, T.J., Mielke, M., Zuniga, J.M., Camic, C. L., Johnson, G.O., Schmidt, R. J. and Housh, D. J. 2010. Acute Effects of a Caffeine-Containing Supplement on Bench Press and Leg Extension Strength and Time to Exhaustion During Cycle Ergometry. Journal of Strength and Conditioning Research, 24(3): 859865.

15. Hudson, G.M., Green, J.M., Bishop, P.A. and Richardson, M.T. 2008. Effects of Caffeine and Aspirin on Light Resistance Training Performance, RPE, and Pain Perception. Journal of Strength and Conditioning Research, 22(6): 1950-1957. 
16. Jacobson, B.H., Weber, M.D., Claypool, L. and Hunt, L.E. 1992. Effect of Caffeine on Maximal Strength and Power in Elite Male Athletes. British Journal of Sports Medicine, 26(4): 276-280.

17. Kamimori, G.H., Lugo, S.T., Penetar, D.M., Chamberlain, A.C., Brunhart, G.E., Brunhart, A.E. and Eddington, N.D. 1995. Dose-dependent caffeine pharmacokinetics during severe sleep deprivation in humans. Int. J. Clin. Pharmacol.Toxicol. Ther, 33 (1): 82-86.

18. Kamimori, G.H., Penetar, D.M. and Headley, D.B. 2000. Effect of three caffeine doses on plasma catecholamines and alertness during prolonged wakefulness. Eur. J.Clin. Pharmacol, 56: 537-44.

19. Kamimori, G.H., Karyekar, C.S., Otterstetter, R., Otterstetter, R., Cox, D.S., Balkin, T.J., Belenky, G.L. \& Eddington, N.D. 2002. The rate of absorption and relative bioavailability of caffeine administered in chewing gum versus capsules to normal healthy volunteers. Int. J. Pharm, 234: 159-67.

20. Meeusen, R., Roelands, B. and Spriet, L.L. 2013. Caffeine, Exercise and the Brain. Limits of Human Endurance, 76: 1-12.

21. Nicolazzo, J.A., Reed, B.L. and Finnin, B.C. 2003. The effect of various in vitro conditions on the permeability characteristics of the buccal mucosa. J. Pharm. Sci, 92: 2399-2410.

22. Paton, C., Lowe, T. and Irvine, A. 2010. Caffeinated chewing gum increases repeated sprint performance and augments increases in testosterone in competitive cyclists. Eur J Appl Physiol, 110: 1243-1250.

23. Pottier, A., Bouckaert, J., Gilis, W., Roels, T. and Derave, W. 2010. Mouth rinse but not ingestion of a carbohydrate solution improves 1-h cycle time trial performance. Scandinavian Journal of Medicine and. Science in Sports, 20: 105-111.

24. Roelands, B., Buyse, L., Pauwels, F., Delbeke, F., Deventer, K. and Meeusen, R. 2011. No effect on exercise perforamance in high ambient temperature. European journal of applied physiology, 111: 3089-3095.

25. Rollo, I., Williams, C., Gant, N. and Nute, M. 2008. The influence of carbohydrate mouth rinse on self-selected speeds during a 30-min treadmill run. International Journal of Sport Nutrition and Exercise Metabolism, 18: 585-60.

26. Ryan, E.J., Kim, C., Fickes, J.E., Williamson, M., Muller, D.M., Barkley, J.E., Gunstad, J. and Glickman, L.E 2013. Caffeine gum and cycling performance: a timing study. Journal of Strength and Conditioning Research, 27(1): 259-264.

27. Sinclair, J., Bottoms, L., Flynn, C., Bradley, E., Alexander, G., McCullagh, S., Finn, T., and Hurst, T. 2014. The effect of different durations of carbohydrate mouth rinse on cycling performance. European Journal of Sports Science, 14(3):259-264.

28. Sokmen, B., Armstrong, L.E., Kraemer, W. J., Casa, D.J., Dias, J.C., Judelson, D.A. and Maresh, C.M. 2008. Caffeine Use in Sports: Considerations for the Athlete. Journal of Strength and Conditioning Research, 22(3): 978-986. 
29. Thakur, R., Meidan, V. and Michniak, B. 2007. Transdermal and buccal delivery of methylxanthines through human tissue in vitro. Drug Develop. \& Ind. Pharmacy, 33: 513-521.

30. Van Soeren, M.H. and Graham, T.E. 1998. Effect of caffeine on metabolism, exercise endurance, and catecholamine responses after withdrawal. Journal of Applied Physiology, 85(4): 1493-1501.

31. Warren, G.L., Park, N.D., Maresca, R.D., McKibans, K.I. and Millard-Stafford, M.L. 2010. Effect of Caffeine Ingestion on Muscular Strength and Endurance: A MetaAnalysis. Medicine \& Science in Sports and Exercise, 42(7): 1375-1387.

32. Woolf, K., Bidwell, W.K. and Carlson, G.A. 2008. The Effect of Caffeine International Journal of Sport Nutrition and Exercise Metabolism, 18: 412 